\title{
Geometria substituta de mama baseada na retirada manual de curvas de termogramas para estimativa de parâmetros termofísicos mamários
}

\author{
José Reginaldo Alves de Queiroz Júnior ${ }^{1}$, Rita de Cássia Fernandes de Lima² \\ ${ }^{1}$ Centro de Ciências Médicas - Universidade Federal de Pernambuco (UFPE) \\ Caixa Postal 50730-120 - Recife - PE - Brasil
}

${ }^{2}$ Departamento de Engenharia Mecânica- Universidade Federal de Pernambuco (UFPE)

Caixa Postal 50670-901 - Recife - PE - Brasil

reginaldoqueirozjr3@gmail.com,ritaflima@yahoo.com

\begin{abstract}
Thermography is a technique has been proposed as an auxiliary tool in the screening of breast cancer. These images are also used for the validation Three-dimensional numerical simulations validation. The production of a more realistic model would allow a reliable estimation of the thermophysical properties of the breast. This work aimed to format a methodology in order to develop a three - dimensional breast geometry from the curves extracted from the thermograms in the UFPE Thermographic Imaging Database.
\end{abstract}

Resumo. A termografia vem sendo proposta como uma ferramenta auxiliar no rastreio do câncer de mama. Essas imagens também são utilizadas na validação de simulações numéricas tridimensionais. A produção de um modelo mais realístico e personalizado possibilitaria uma estimativa mais precisa das propriedades termofísicas da mama. Este trabalho teve como objetivo o desenvolvimento de uma metodologia para a produção de uma geometria tridimensional personalizada da mama a partir das curvas extraídas dos termogramas contidos no Banco de Dados de Imagens Termográficas da UFPE.

\section{Introdução}

O envelhecimento e a mudança dos tipos de doenças da população, as transições demográficas e epidemiológicas, respectivamente, sinalizam um impacto cada vez maior da carga de doenças crônicas não-transmissíveis (DCNT) nas próximas décadas [Duarte e Barreto, 2012]. Atualmente, no Brasil, doenças cardiovasculares e neoplasias, ambas DCNT, ainda são as principais causas de mortes [Schimidt, Duncan, e Stevens 2011].

Para 2018 as estimativas realizadas pelo Instituto Nacional de Câncer apontam a ocorrência de 59.700 novos casos de câncer de mama entre mulheres. O Brasil é um país com grande variação da taxa de frequência desse tipo de neoplasia. Esse tipo de câncer é o primeiro mais frequente nas mulheres da Região Nordeste $(40,36 / 100$ mil) [INCA, 2017].

Atualmente, a mamografia é o exame de imagem de primeira escolha para a detecção do câncer de mama, mas apresenta limitações. Costuma ser menos sensível na detecção de tumores em mulheres com tecido mamário denso. Devido à compressão durante a realização da mamografia, existe o risco de ruptura do encapsulamento do tumor [Ramírez-Torres et al., 2017]. 
A termografia é menos desconfortável, pois não implica em contato direto, não usa radiação ionizante nem compressão da mama. É útil para mulheres de todas as idades e em condições diferentes, como gravidez, implantes, seios densos ou fibrocísticos [Avila-Castro et al., 2017]. A detecção do câncer é justificada pela neoangiogênese causada pela neoplasia, que pode provocar um aumento na temperatura na região próxima ao tumor [Ramírez-Torres et al., 2017].

A termografia também vem sendo utilizada para validar simulações numéricas de perfis de temperatura e para estimativas de parâmetros termofísicos [Bezerra et al.,2013]. À medida que as imagens médicas são capazes de fornecer uma série de informações clínicas relevantes, a modelagem fisiológica pode ser uma ferramenta preditiva auxiliar, na compreensão dos processos físicos e biológicos [Avila-Castro et al., 2017]. O aprimoramento de técnicas que contribuam para o rastreamento do câncer é uma área da medicina que pode se beneficiar com o uso de tais ferramentas.

A proposta desse trabalho é o desenvolvimento de uma geometria substituta personalizada a partir da extração manual de curvas contidas nos termogramas indexados no Banco de Dados de Imagens Termográficas da UFPE. Em seguida, foram realizados cálculos de perfis de temperatura com a geometria tridimensional da mama desenvolvida.

\section{Metodologia}

Todas a investigações fazem parte de um projeto intitulado "Análise da viabilidade do uso de câmera termográfica como ferramenta auxiliar no diagnóstico de câncer de mama em hospital público localizado em clima tropical" registrado no Ministério da Saúde (CEP/CCS/UFPE n²79/05). As imagens por infravermelho (IR) utilizadas foram obtidas a partir da utilização de uma câmera de infravermelho FLIR S45, adquirida pela FINEP e alocada no Dpto. de Engenharia Mecânica da UFPE. A confiabilidade na aquisição e o processamento adequado das imagens dependem da padronização conveniente dos processos.

\subsection{Aquisição de imagens}

O procedimento foi realizado em uma sala do HC/UFPE. Durante a aquisição das imagens são realizadas duas séries de imagens, a primeira (D1) a uma distância fixa, com a câmera posicionada mais longe da paciente. A segunda série (D2) é realizada com a câmera mais próxima da paciente, com distância variável. Todo o processo de aquisição encontra-se detalhado em Oliveira (2012).

\subsection{Extração da curva termográfica}

Em exames mais recentes, algumas IR foram realizadas com o auxílio de uma grade metálica (quadrados com arestas de $75 \mathrm{~mm}$ ) o que permitiu obter a dimensão do eixo horizontal $(\mathrm{MH})$ para ajuste da base que representa a parede torácica da paciente, conforme as Figuras 1(a) e 1(b) e utilizando a equação abaixo:

$$
M H_{\text {real }}=\frac{M H(\mathrm{~mm}) \times 7,5(\mathrm{~cm})}{M Q(\mathrm{~mm})}
$$

onde MH é medida horizontal extraída manualmente, MQ é medida da grade extraída manualmente e 7,5 centímetros é a medida real da grade. 


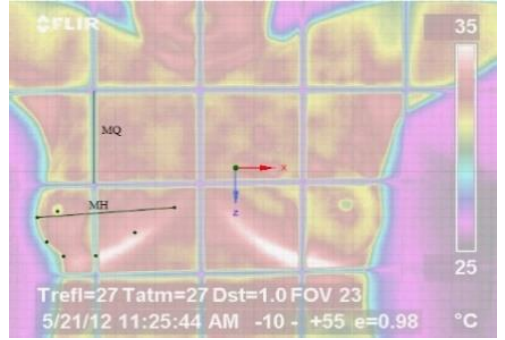

(a)

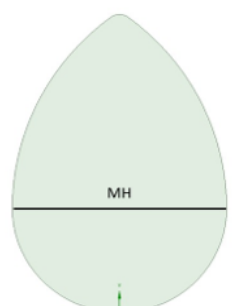

(b)

Figura 1 - (a) Vista frontal de termograma, com destaque para Medida Horizontal (MH) e Medida Quadrado (MQ); (b) Base que representa a base da mama, com destaque para a Medida Horizontal (MH).

A extração manual da curva de perfil da mama foi realizada através do software SpaceClaim $^{\circledR}$, incluso no pacote de softwares de CFD (Computational Fluid Dynamics) do ANSYS. Com a escolha dos pontos na região limite da mama, a curva de perfil foi construída ponto a ponto, o que permitiu um ajuste final para sua suavização (Figura 2).

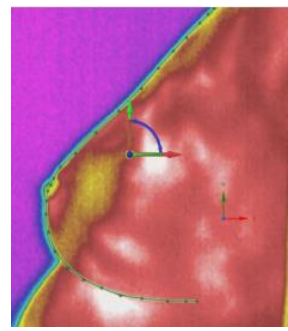

Figura 2 - Extração manual da curva de perfil de um termograma.

\subsection{Modelagem da geometria substituta}

Foi efetuada a adequação da curva de perfil à parede torácica, assumindo seu tamanho real proporcional à reta $\mathrm{MH}$. Essa fase foi responsável pelo ajuste de uma curva que parte de um dos extremos da reta $\mathrm{MH}$, cruzando o ponto que simboliza o mamilo no perfil e fechando seu percurso na outra extremidade dessa mesma reta, como demonstrado na Fig.3b. Para inserir o nódulo na geometria construída, foram utilizados os dados fornecidos pelo exame de ultrassonografia da paciente, com a origem do eixo de coordenadas no mamilo, visto que este é o local de referência adotado pelo exame clínico.

A Figura 3 a mostra as etapas propostas para geração dos volumes personalizados. Na mesma, são visualizados contornos extraídos da termografia de uma paciente e o uso de uma elipse escolhida para suavizar a curva frontal da mama.

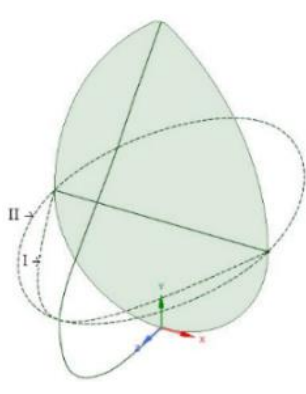

(a)

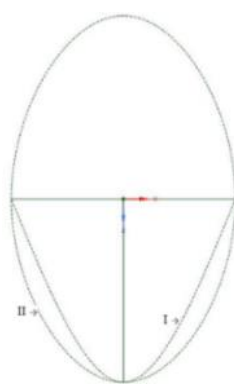

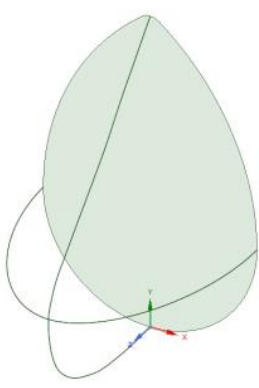

(b)

Figura 3 - (a) Comparação entre Curvas I e II para ajuste necessário na construção da geometria; (b) Curva de perfil e base ajustadas para construção de geometria. 
Viana (2010) utilizou uma geometria um pouco mais realista em seu trabalho, no entanto, fez uso de cinco software, o que dificulta o objetivo final. Além disso, em alguns casos, foram observadas divergências entre a geometria final e a mama da paciente. A metodologia adotada pelo presente trabalho proporciona uma simplificação do processo anterior, por usar um número menor de ferramentas computacionais. E, ao extrair manualmente a curva de perfil, a geometria final tende a ser mais próxima da mama da paciente analisada.

\section{Resultados e Discussões}

Nesta seção são descritas as geometrias geradas pela modelagem proposta para duas pacientes, uma com uma neoplasia maligna e outra com uma neoplasia benigna. As temperaturas obtidas pelas simulações numéricas foram comparadas com os valores obtidos por Bezerra et al. (2013) e com os valores medidos pela termografia.

\subsection{Estudo de Caso 1}

Paciente 1, sexo feminino, 49 anos, diagnosticada com nódulo sólido na mama esquerda, BI-RADS V, confirmado pelos exames de mamografia e ultrassonografia. O tumor está situado no quadrante superior lateral (QSL), com dimensões de $17 \times 8 \times 17 \mathrm{~mm}$ e a 16 $\mathrm{mm}$ de profundidade da pele. O nódulo teve sua geometria aproximada para um cilindro com bases elípticas e taxa de calor metabólico calculado (seguindo as equações descritas em Bezerra et al., 2013) de $25.848 \mathrm{~W} / \mathrm{m}^{3}$.

A aproximação da geometria do tumor para um cilindro de base elipsoidal foi estabelecida como forma de melhor utilizar os dados que são obtidos pelos exames de imagem da ultrassonografia, que costumam fornecer as informações dos tumores com base nas três dimensões, além da sua localização em relação à pele e ao mamilo.

Seguindo a metodologia proposta, essa paciente possui o eixo horizontal medindo $117 \mathrm{~mm}$. Baseado neste valor e na sua curva de perfil, a geometria da mama e do nódulo da paciente foram construídas. Para a solução numérica do problema, foi realizado um estudo de convergência da malha, testando-se dez malhas tetraédricas não estruturadas distintas. Para este caso, foi então escolhida uma malha de 301.826 nós.

A Figura 4 mostra duas geometrias geradas para a paciente deste Estudo de caso, uma gerada pelo método desenvolvido por Viana (2010) (a) e outra produzida pelo método desenvolvido neste trabalho (b). A Tabela 1 mostra o volume de cada geometria tridimensional. A diferença percentual entre eles é de 113,6\%.

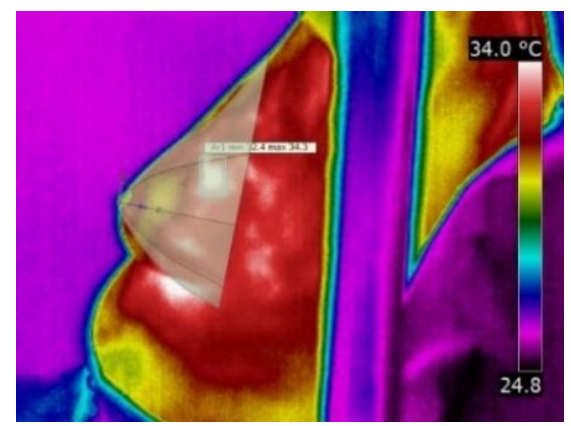

(a)

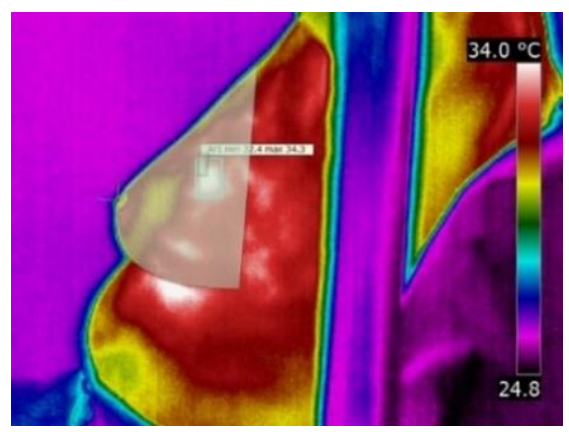

(b)

Figura 23 - (a) Geometria produzida por Viana (2010) para a paciente do Estudo de caso 1; (b) Geometria desenvolvida neste trabalho para a paciente do Estudo de caso 1. 
Nesse Estudo de Caso, o valor medido pela imagem termográfica é igual a 35,27 ${ }^{\circ} \mathrm{C}$. A Tabela 2 resume os resultados obtidos (temperatura máxima) em cada análise. Para o Estudo de Caso 1, o erro entre o valor calculado numericamente por Bezerra (2013) e a medição da câmera termográfica foi de $0,169 \%$, enquanto que o mesmo erro calculado pelo presente trabalho foi de $0,085 \%$.

\subsection{Estudo de Caso 2}

Paciente 2, sexo feminino, 27 anos, submeteu-se a exame clínico, ultrassonografia, mamografia e biópsia com diagnóstico de fibroadenoma na mama esquerda. Tumor situado no quadrante inferior lateral (QIL), com dimensões de 23 × 15 × $23 \mathrm{~mm}$ e a $9 \mathrm{~mm}$ de profundidade da pele. O nódulo foi aproximado para um cilindro de bases elípticas e a taxa de calor metabólico calculada de $8.835,36 \mathrm{~W} / \mathrm{m}^{3}$. Essa paciente possui o eixo horizontal medindo $121 \mathrm{~mm}$. Baseado neste valor e na sua curva de perfil, a geometria da mama e do nódulo da paciente foram construídas. Foram testadas dez malhas tetraédricas não estruturadas distintas e escolhida uma malha de 380.525 nós.

A Figura 5 mostra as duas geometrias geradas para a paciente deste estudo de caso, uma gerada pelo método desenvolvido por Viana (2010) (a) e outra produzida pelo método desenvolvido neste trabalho (b). A Tabela 1 mostra o volume de cada geometria tridimensional. A diferença percentual entre eles é de $22,7 \%$.

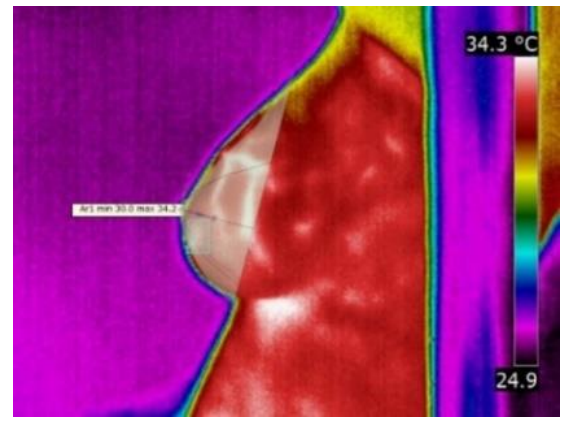

(a)

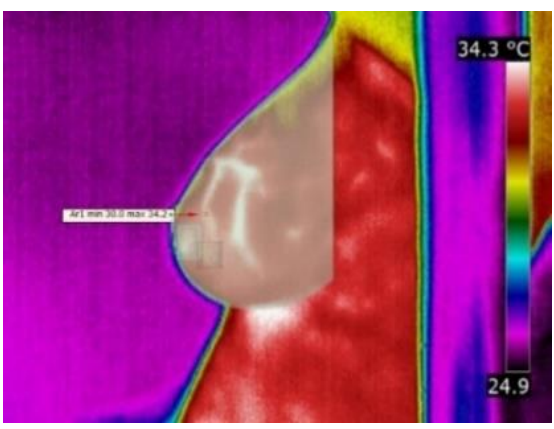

(b)

Figura 27 - (a) Geometria produzida por Viana (2010) para a paciente do Estudo de caso 2; (b) Geometria desenvolvida neste trabalho para a paciente do Estudo de caso 2.

Tabela 1. Comparação dos volumes obtidos nos Estudos de Caso

\begin{tabular}{|c|c|c|}
\hline Método & Estudo de Caso 1 & Estudo de Caso 2 \\
\hline Viana (2010) & $1,63 \mathrm{dm}^{3}$ & $0,70 \mathrm{dm}^{3}$ \\
\hline Presente trabalho & $0,76 \mathrm{dm}^{3}$ & $0,60 \mathrm{dm}^{3}$ \\
\hline
\end{tabular}

Para esse Estudo de Caso, o valor medido pela imagem termográfica é igual a $35,20{ }^{\circ} \mathrm{C}$. Assim, o erro entre o valor calculado numericamente por Bezerra (2013) e a medição da câmera termográfica foi de $0,060 \%$, enquanto que o mesmo erro calculado pelo presente trabalho foi de $0,00 \%$.

Tabela 2. Comparação das temperaturas máximas obtidas nos Estudos de Caso

\begin{tabular}{|c|c|c|}
\hline Método & Estudo de Caso 1 & Estudo de Caso 2 \\
\hline Bezerra $(2013)$ & $35,33^{\circ} \mathrm{C}$ & $35,18^{\circ} \mathrm{C}$ \\
\hline Presente trabalho & $35,30^{\circ} \mathrm{C}$ & $35,20^{\circ} \mathrm{C}$ \\
\hline
\end{tabular}


A diferença entre os resultados obtidos por Bezerra (2013) e por este trabalho é atribuída, sobretudo, à nova geometria utilizada para o cálculo das temperaturas superficiais confirmando que quanto mais próxima da geometria real, melhores serão os resultados obtidos, por meio de simulações numéricas computacionais.

\section{Conclusão}

A metodologia sugerida por este trabalho trouxe uma simplificação do processo de produção de modelos tridimensionais da mama baseados em imagens termográficas. Com a extração manual da curva de perfil, a geometria 3D final apresenta um aspecto muito mais semelhante com a anatomia superficial da mama feminina. Os resultados obtidos pela simulação numérica do perfil de temperatura foram comparados com os resultados obtidos usando-se geometria anterior, gerada por Viana (2010), mostrando que a metodologia proposta no presente trabalho pode ser utilizada com sucesso para os cálculos mencionados. Além disso, o novo processo eliminou o uso de outros softwares desenvolvidos por outros autores, simplificando o procedimento de aquisição das curvas da mama necessárias ao desenvolvimento do volume tridimensional e reduzindo a propagação de possíveis erros inerentes ao processo.

\section{References}

Avila-Castro, I. A.; Hernández-Martínez, A.R.; Estevez, M.; Cruz, M.; Esparza, R.; Pérez, R.; Rodríguez, A.L. (2017) Thorax thermographic simulator for breast pathologies. Journal of Applied Research and Technology, v. 15, n. 2, p. 143-151.

Bezerra, L. A., Oliveira, M.M.; Rolim, T.L.; Conci, A.; Santos, F.G.S.; Lyra, P.R.M.; Lima, R.C.F. (2013) Estimation of breast tumor thermal properties using infrared images. Signal Processing, v. 93, n. 10, p. 2851-2863.

Duarte, E. C.; Barreto, S.M. (2012) Transição demográfica e epidemiológica: a Epidemiologia e Serviços de Saúde revisita e atualiza o tema. Epidemiol. Serv. Saúde. Brasília, v. 21, n. 4, p. 529-532.

INCA. Instituto Nacional de Câncer José Alencar Gomes da Silva. (2017) Estimativas 2018: Incidência de Câncer no Brasil. Rio de Janeiro, Brasil: Ministério da Saúde.

Oliveira, M. (2012) Desenvolvimento de protocolo e construção de um aparato mecânico para padronização da aquisição de imagens termográficas de mama. 2012. 104p. Dissertação (Mestrado em Engenharia Mecânica) - Universidade Federal de Pernambuco, Recife.

Ramírez-Torres, A.; Rodríguez-Ramos, R.; Sabina, F.J.; García-Reimbert, C.; Penta, R.; Merodio, J.; Guinovart-Díaz, R.; Bravo-Castillero, J.; Conci, A.; Preziosi, L. (2017) The role of malignant tissue on the thermal distribution of cancerous breast. Journal of Theoretical Biology, v. 426, p. 152-161.

Schmidt, I.; Duncan, B.; Stevens, A. (2011) Doenças crônicas não transmissíveis no Brasil: carga e desafios atuais. In: Victora CG et al. Saúde no Brasil: a série The Lancet. Rio de Janeiro: Fiocruz; p. 61-74.

Viana, M.J.A. (2010) Simulação do perfil de temperatura na mama através de geometria substituta obtida a partir de prótese mamária externa. 2010. 118p. Dissertação (Mestrado em Engenharia Mecânica) - Universidade Federal de Pernambuco, Recife. 